\title{
THE CAUCHY PROBLEM AND DECAY RATES \\ FOR STRONG SOLUTIONS OF A BOUSSINESQ SYSTEM
}

\author{
FRANCISCO GUILLÉN GONZÁLEZ, MÁRCIO SANTOS DA ROCHA, \\ AND MARKO ROJAS MEDAR
}

Received 10 June 2004

The Boussinesq equations describe the motion of an incompressible viscous fluid subject to convective heat transfer. Decay rates of derivatives of solutions of the three-dimensional Cauchy problem for a Boussinesq system are studied in this work.

\section{Introduction}

In this work we show some theoretical results about decay rates of strong solutions of the three-dimensional Cauchy problem for Boussinesq equations, described by the following partial differential equation problem (see [6]):

$$
\begin{gathered}
\frac{\partial \mathbf{u}}{\partial t}-\nu \triangle \mathbf{u}+\mathbf{u} \cdot \nabla \mathbf{u}+\nabla \pi=\theta \mathbf{f} \quad \text { in }(0, T) \times \mathbb{R}^{3}, \\
\operatorname{div} \mathbf{u}=0 \quad \text { in }(0, T) \times \mathbb{R}^{3}, \\
\frac{\partial \theta}{\partial t}-\chi \triangle \theta+\mathbf{u} \cdot \nabla \theta=0 \quad \text { in }(0, T) \times \mathbb{R}^{3}, \\
\mathbf{u}(0, x)=\mathbf{a}(x) \quad \text { in } \mathbb{R}^{3}, \\
\theta(0, x)=b(x) \quad \text { in } \mathbb{R}^{3},
\end{gathered}
$$

where the unknown are $\mathbf{u}, \theta, \pi$ which denote, respectively, the velocity field, the scalar temperature and the scalar pressure. Data are the positive constants $\nu, \chi$, respectively, the viscosity and the thermal conductivity coefficients and the function $\mathbf{f}$ the external force field, and $\mathbf{a}(x), b(x)$, respectively, represent the initial velocity and initial temperature.

The main objective of this work is to obtain a decay rate of derivatives for the strong solutions to the Cauchy problem (1.1). For this, we will consider the usual Lebesgue spaces $L^{p}\left(\mathbb{R}^{3}\right)$ with the usual norms $|\cdot|_{p}$. We will denote $L_{\sigma}^{p}\left(\mathbb{R}^{3}\right)$ the closure of $C_{0, \sigma}^{\infty}\left(\mathbb{R}^{3}\right)=$ $\left\{\mathbf{v} \in C_{0}^{\infty} ; \operatorname{div} \mathbf{v}=0\right\}$ in $L^{p}\left(\mathbb{R}^{3}\right)$. We will denote too by $L^{P}\left(0, T ; L^{q}\left(\mathbb{R}^{3}\right)\right)$ the Banach space, classes of functions defined a.e. in $[0, T]$ on $L^{q}\left(\mathbb{R}^{3}\right)$, that are $L^{p}$-integrable in the sense of Bochner. For more details see $[1,3]$.

We observe that this model of fluids includes as a particular case the classical NavierStokes equations, which has been thoroughly studied (see, e.g., [7, 8]). Rojas Medar and 
Lorca obtained results of uniqueness and existence of the local solutions and regularity of solutions for Boussinesq equations $[9,10]$.

Results of decay rates of strong solution were obtained by Cheng He and Ling Hsião [4]. In this paper, we are interested to get similar results for Boussinesq equations.

\section{Results of decay rates}

The main objective of this work is to establish the decay rates of derivatives about time variable and spaces variables for the strong solutions to the Cauchy problem of the Boussinesq equations (1.1). For this, we will consider a sequence of Cauchy problems for the linearized Boussinesq equations

$$
\begin{gathered}
\frac{\partial \mathbf{u}^{k}}{\partial t}-v \triangle \mathbf{u}^{k}+\left(\mathbf{u}^{k-1} \cdot \nabla\right) \mathbf{u}^{k-1}+\nabla \pi^{k}=\theta^{k-1} \mathbf{f} \quad \text { on }(0, T) \times \mathbb{R}^{3}, \\
\operatorname{div} \mathbf{u}^{k}=0 \quad \text { on }(0, T) \times \mathbb{R}^{3}, \\
\frac{\partial \theta^{k}}{\partial t}-\chi \triangle \theta^{k}+\mathbf{u}^{k-1} \cdot \nabla \theta^{k-1}=0 \quad \text { on }(0, T) \times \mathbb{R}^{3}, \\
\mathbf{u}^{k}(0, x)=\mathbf{a}^{k}(x) \quad \text { on } \mathbb{R}^{3}, \\
\theta^{k}(0, x)=b^{k}(x) \text { on } \mathbb{R}^{3},
\end{gathered}
$$

for $k \geq 1$, where $\mathbf{a}^{k} \in C_{0, \sigma}^{\infty}\left(\mathbb{R}^{3}\right)$ and $b^{k} \in C_{0}^{\infty}\left(\mathbb{R}^{3}\right)$ such that

$$
\begin{array}{ll}
\mathbf{a}^{k} \longrightarrow \mathbf{a} & \text { in } L_{\sigma}^{3}\left(\mathbb{R}^{3}\right) \text { strongly, } \\
b^{k} \longrightarrow b & \text { in } L^{3}\left(\mathbb{R}^{3}\right) \text { strongly, }
\end{array}
$$

with $\left|\mathbf{a}^{k}\right|_{3} \leq|\mathbf{a}|_{3}$ and $\left|b^{k}\right|_{3} \leq|b|_{3}$. The first term, $\left(\mathbf{u}^{0}, \pi^{0}, \theta^{0}\right)$, of this sequence is solution of the trivial Cauchy problem:

$$
\begin{gathered}
\frac{\partial \mathbf{u}^{0}}{\partial t}-v \triangle \mathbf{u}^{0}+\nabla \pi^{0}=\mathbf{0} \quad \text { on }(0, T) \times \mathbb{R}^{3} \\
\operatorname{div} \mathbf{u}^{0}=0 \quad \text { on }(0, T) \times \mathbb{R}^{3} \\
\frac{\partial \theta^{0}}{\partial t}-\chi \triangle \theta^{0}=0 \quad \text { on }(0, T) \times \mathbb{R}^{3} \\
\mathbf{u}^{0}(0, x)=\mathbf{a}^{0}(x) \quad \text { on } \mathbb{R}^{3} \\
\theta^{0}(0, x)=b^{0}(x) \quad \text { on } \mathbb{R}^{3}
\end{gathered}
$$

Let $\Gamma_{\nu}(t, x ; s, y)=(4 v \pi t)^{-3 / 2} \exp \left(-|x|^{2} / 4 v t\right)$ be a fundamental solution of the heat equation in $\mathbb{R}^{3}$ (with viscosity coefficient $\nu$ ). Then, the solution of the linearized Boussinesq 
system (2.1) can be written as follows:

$$
\begin{aligned}
u_{i}^{k}(t, x)= & \int_{\mathbb{R}^{3}} \Gamma_{\nu}(t, x ; 0, y) a_{i}^{k}(y) d y \\
& -\int_{0}^{t} \int_{\mathbb{R}^{3}} \Gamma_{\nu}(t, x ; s, y) \sum_{j=1}^{3} u_{j}^{k-1}(s, y) \frac{\partial u_{i}^{k-1}}{\partial x_{j}}(s, y) d y d s \\
& -\int_{0}^{t} \int_{\mathbb{R}^{3}} \Gamma_{\nu}(t, x ; s, y) \frac{\partial \pi^{k}}{\partial x_{i}}(s, y) d y d s \\
& +\int_{0}^{t} \int_{\mathbb{R}^{3}} \Gamma_{\nu}(t, x ; s, y) \theta^{k-1}(s, y) f_{i}(s, y) d y d s, \\
\theta^{k}(t, x)= & \int_{\mathbb{R}^{3}} \Gamma_{\chi}(t, x ; 0, y) b^{k}(y) d y \\
& -\int_{0}^{t} \int_{\mathbb{R}^{3}} \Gamma_{\chi}(t, x ; s, y) \sum_{j=1}^{3} u_{j}^{k-1}(s, y) \frac{\partial \theta^{k-1}}{\partial x_{j}}(s, y) d y d s .
\end{aligned}
$$

The convergence for the above method can be seen in $[2,7]$.

Definition 2.1. A couple $(\mathbf{u}, \theta)$ is called strong solution for the system (1.1), if

$$
\begin{aligned}
& \mathbf{u} \in L^{p}\left(0, \infty ; L_{\sigma}^{q}\left(\mathbb{R}^{3}\right)\right) \cap L^{\infty}\left(0, \infty ; L_{\sigma}^{3}\left(\mathbb{R}^{3}\right)\right), \\
& \theta \in L^{p}\left(0, \infty ; L^{q}\left(\mathbb{R}^{3}\right)\right) \cap L^{\infty}\left(0, \infty ; L^{3}\left(\mathbb{R}^{3}\right)\right)
\end{aligned}
$$

for some $p>2$ and $q>3$, and satisfying

$$
\begin{array}{r}
\int_{0}^{\infty} \int_{\mathbb{R}^{3}}\left(\mathbf{u} \cdot \frac{d \varphi}{d t}+\mathbf{u} \cdot \Delta \varphi+(\mathbf{u} \cdot \nabla \varphi) \mathbf{u}\right) d x d t \\
=-\int_{\mathbb{R}^{3}} \mathbf{a} \cdot \varphi(0, x) d x+\int_{0}^{\infty} \int_{\mathbb{R}^{3}} \theta \mathbf{f} \cdot \varphi d x d t
\end{array}
$$

for all $\varphi \in C_{0}^{\infty}\left(0, \infty ; C_{0, \sigma}^{\infty}\left(\mathbb{R}^{3}\right)\right)$ and

$$
\int_{0}^{\infty} \int_{\mathbb{R}^{3}}\left(\theta \frac{d \psi}{d t}+\theta \Delta \psi+(\mathbf{u} \cdot \nabla \psi) \theta\right) d x d t=-\int_{\mathbb{R}^{3}} b \psi(0, x) d x
$$

for all $\psi \in C_{0}^{\infty}\left(0, \infty ; C_{0}^{\infty}\left(\mathbb{R}^{3}\right)\right)$.

Lemma 2.2. For the pressure $\pi^{k}$ the following estimate holds:

$$
\left|\nabla \pi^{k}\right|_{r} \leq C\left|\left(\mathbf{u}^{k-1} \cdot \nabla\right) \mathbf{u}^{k-1}+\theta^{k-1} \mathbf{f}\right|_{r}
$$

for $1<r<\infty, k>0$. 
Lemma 2.3. Let $\mathbf{a} \in L_{\sigma}^{3}\left(\mathbb{R}^{3}\right), b \in L^{3}\left(\mathbb{R}^{3}\right), \quad|\mathbf{f}(\mathbf{t})|_{q} \leq C_{0} t^{-1+3 / 2 q}\left(|\mathbf{a}|_{3}+|b|_{3}\right), \quad|\nabla \mathbf{f}(t)|_{q} \leq$ $C_{0} t^{-3 / 2+3 / 2 q}\left(|\mathbf{a}|_{3}+|b|_{3}\right)$, where the constant $C_{0}$ is independent of $t$ and $q$. If $C^{*} C_{0}\left(|\mathbf{a}|_{3}+\right.$ $\left.|b|_{3}\right) \leq 1$ for some constant $C^{*}$, then

$$
\begin{aligned}
t^{1 / 2-3 / 2 q}\left(\left|\mathbf{u}^{k}(t)\right|_{q}+\left|\theta^{k}(t)\right|_{q}\right) & \leq C C_{0}\left(|\mathbf{a}|_{3}+|b|_{3}\right), \\
t^{1-3 / 2 q}\left(\left|\nabla \mathbf{u}^{k}(t)\right|_{q}+\left|\nabla \theta^{k}(t)\right|_{q}\right) & \leq C C_{0}\left(|\mathbf{a}|_{3}+|b|_{3}\right)
\end{aligned}
$$

for $3 \leq q \leq \infty, t \geq 0$ and $k \geq 0$.

Proof. We put

$$
\begin{aligned}
& I_{q}^{k}=t^{1 / 2-3 / 2 q}\left(\left|\mathbf{u}^{k}(t)\right|_{q}+\left|\theta^{k}(t)\right|_{q}\right) \\
& J_{q}^{k}=t^{1-3 / 2 q}\left(\left|\nabla \mathbf{u}^{k}(t)\right|_{q}+\left|\nabla \theta^{k}(t)\right|_{q}\right) .
\end{aligned}
$$

We will assume by inductive hypotheses that the estimates (2.10) are true for $k-1$. By the Young inequality for convolution, we can estimate the terms of (2.4) as follows:

$$
\begin{aligned}
\left|\int_{\mathbb{R}^{3}} \Gamma_{\nu}(t, x ; 0, y) a_{i}^{k}(y) d y\right|_{q} & \leq(4 v \pi t)^{-3 / 2}\left(\int_{\mathbb{R}^{3}} e^{-|x-y|^{2} / 4 v t} d y\right)^{1 / p}\left|a_{i}^{k}\right|_{3} \\
& \leq C t^{-1 / 2+3 / 2 q}\left|a_{i}^{k}\right|_{3},
\end{aligned}
$$

where $1 / p+1 / 3=1+1 / q$. Again, by the Young inequality we obtain

$$
\begin{aligned}
& \left|\int_{0}^{t} \int_{\mathbb{R}^{3}} \Gamma_{\nu}(t, x ; s, y) \sum_{j=1}^{3} u_{j}^{k-1}(s, y) \frac{\partial u_{i}^{k-1}}{\partial x_{j}}(s, y) d y d s\right|_{q} \\
& \quad \leq C \int_{0}^{t}(t-s)^{-3 / 2(1 / 2-1 / q)}\left|\mathbf{u}^{k-1}\right|_{4}\left|\nabla \mathbf{u}^{k-1}\right|_{4} d s \\
& \leq C C_{0}^{2}\left(|\mathbf{a}|_{3}+|b|_{3}\right)^{2} t^{-1 / 2+3 / 2 q},
\end{aligned}
$$

where $1 / p+1 / 2=1+1 / q$. Now, using (2.9) we have

$$
\begin{gathered}
\left|\int_{0}^{t} \int_{\mathbb{R}^{3}} \Gamma_{\nu}(t, x ; s, y) \frac{\partial \pi^{k}}{\partial x_{i}}(s, y) d y d s\right|_{q} \leq C C_{0}^{2}\left(|\mathbf{a}|_{3}+|b|_{3}\right)^{2} t^{-1 / 2+3 / 2 q}, \\
\left|\int_{0}^{t} \int_{\mathbb{R}^{3}} \Gamma_{\nu}(t, x ; s, y) \theta^{k-1}(s, y) f_{i}(s, y) d y d s\right|_{q} \leq C C_{0}^{2} t^{-1+3 / 2 q}\left(|\mathbf{a}|_{3}+|b|_{3}\right)^{2} .
\end{gathered}
$$

Moreover

$$
\left|\mathbf{u}^{k}(t)\right|_{q} \leq C t^{-1 / 2+3 / 2 q}\left(C_{0}\left(|\mathbf{a}|_{3}+|b|_{3}\right)+C_{0}^{2}\left(|\mathbf{a}|_{3}+|b|_{3}\right)^{2}\right) .
$$

Analogously, we can obtain the estimate for $\left|\theta^{k}(t)\right|_{q}$. Now, differentiating (2.4) and using the fact

$$
\left|\frac{\partial}{\partial x_{l}} \Gamma_{\nu}(t, x ; s, y)\right| \leq C(t-s)^{-2} e^{-\lambda|x-y|^{2} / 4 v(t-s)}
$$


for $i=1,2,3$ and some constant $\lambda>0$, follows

$$
\left|\frac{\partial}{\partial x_{l}} \int_{\mathbb{R}^{3}} \Gamma_{\nu}(t, x ; 0, y) a_{i}^{k}(y) d y\right|_{q} \leq C t^{-1+3 / 2 q}|\mathbf{a}|_{3}
$$

Analogously, we obtain

$$
\begin{aligned}
& \left|\frac{\partial}{\partial x_{l}} \int_{0}^{t} \int_{\mathbb{R}^{3}} \Gamma_{\nu}(t, x ; s, y) \sum_{j=1}^{3} u_{j}^{k-1}(s, y) \frac{\partial u_{i}^{k-1}}{\partial x_{j}}(s, y) d y d s\right|_{q} \\
& \quad \leq C C_{0}^{2}\left(|\mathbf{a}|_{3}+|b|_{3}\right)^{2} t^{-1+3 / 2 q} \\
& \left.\quad \frac{\partial}{\partial x_{i}} \int_{0}^{t} \int_{\mathbb{R}^{3}} \Gamma_{\nu}(t, x ; s, y) \frac{\partial \pi^{k}}{\partial x_{i}}(s, y) d y d s\right|_{q} \\
& \quad \leq C C_{0}^{2}\left(|\mathbf{a}|_{3}+|b|_{3}\right)^{2} t^{-1+(3 / 2 q)} \int_{0}^{1}(1-w)^{-1 / 2-3 / 2 r} w^{-3 / 2+3 / 2(1 / r+1 / q)} d w,
\end{aligned}
$$

where $r>3$ and $1 / r+1 / q>1 / 3$, to obtain the convergence of the last integral in (2.19). Finally, we obtain

$$
\left|\frac{\partial}{\partial x_{i}} \int_{0}^{t} \int_{\mathbb{R}^{3}} \Gamma_{\nu}(t, x ; s, y) \theta^{k-1} f_{i} d y d s\right|_{q} \leq C \int_{0}^{t}(t-s)^{-1 / 2+3 / 2(1 / q-1 / l)}\left|\theta^{k-1} f_{i}\right|_{l} d s .
$$

Without difficulty we can obtain for the equation of the temperature

$$
J_{q}^{k} \leq C_{0}\left(|\mathbf{a}|_{3}+|b|_{3}\right)+C C_{0}^{2}\left(|\mathbf{a}|_{3}+|b|_{3}\right)^{2} \leq 2 C_{0}\left(|\mathbf{a}|_{3}+|b|_{3}\right)
$$

For $q=\infty$ we can obtain analogously as before

$$
\begin{aligned}
I_{\infty}^{k} \leq & C\left(|\mathbf{a}|_{3}+|b|_{3}\right)+C t^{1 / 2} \int_{0}^{t}(t-s)^{-3 / 4}\left|\mathbf{u}^{k-1}\right|_{4}\left(\left|\nabla \mathbf{u}^{k-1}\right|_{4}+\left|\nabla \theta^{k-1}\right|_{4}\right) d s \\
& +C t^{1 / 2} \int_{0}^{t}(t-s)^{-3 / 4}\left|\theta^{k-1}\right|_{4}|\mathbf{f}|_{4} d s \leq C\left(|\mathbf{a}|_{3}+|b|_{3}\right)+C C_{0}^{2}\left(|\mathbf{a}|_{3}+|b|_{3}\right)^{2} .
\end{aligned}
$$

Similarly, we obtain the estimative for $J_{\infty}^{k}$.

Lemma 2.4. Let $\mathbf{a} \in L_{\sigma}^{3}\left(\mathbb{R}^{3}\right), \quad b \in L^{3}\left(\mathbb{R}^{3}\right)$ and $|\mathbf{f}|_{q} \leq C_{0} t^{-1+3 / 2 q}\left(|\mathbf{a}|_{3}+|b|_{3}\right), \quad|\nabla \mathbf{f}|_{q} \leq$ $C_{0} t^{-3 / 2+3 / 2 q}\left(|\mathbf{a}|_{3}+|b|_{3}\right)$. If $C^{*} C_{0}\left(|\mathbf{a}|_{3}+|b|_{3}\right) \leq 1$ for some constant $C^{*}$, then for $3 \leq q \leq \infty$, the following estimate is true uniformly in $k$ :

$$
t^{3 / 2-3 / 2 q} \sum_{l, j=1}^{3}\left(\left|\frac{\partial^{2} \mathbf{u}^{k}}{\partial x_{l} \partial x_{j}}\right|_{q}+\left|\frac{\partial^{2} \theta^{k}}{\partial x_{l} \partial x_{j}}\right|_{q}\right) \leq 2 C_{0}\left(|\mathbf{a}|_{3}+|b|_{3}\right)
$$


Proof. The identity (2.4) can be written as follows:

$$
\begin{aligned}
u_{i}^{k}(t, x)= & \int_{\mathbb{R}^{3}} \Gamma_{\nu}(t, x ; 0, y) a_{i}^{k}(y) d y-\int_{0}^{t / 2} \int_{\mathbb{R}^{3}} \Gamma_{\nu}(t, x ; s, y) \\
& \times\left(\sum_{j=1}^{3} u_{j}^{k-1}(s, y) \frac{\partial u_{i}^{k-1}}{\partial x_{j}}(s, y)+\frac{\partial \pi^{k}}{\partial x_{i}}(s, y)-\theta^{k-1}(s, y) f_{i}(s, y)\right) d y d s \\
& -\int_{t / 2}^{t} \int_{\mathbb{R}^{3}} \Gamma_{\nu}(t, x ; s, y)\left(\sum_{j=1}^{3} u_{j}^{k-1}(s, y) \frac{\partial u_{i}^{k-1}}{\partial x_{j}}(s, y)\right. \\
& \left.+\frac{\partial \pi^{k}}{\partial x_{i}}(s, y)-\theta^{k-1}(s, y) f_{i}(s, y)\right) d y d s
\end{aligned}
$$

analogously for temperature.

We will make the case $l=j$ (for the case $l \neq j$ the argument is analogous). By the Young inequality we obtain

$$
\begin{aligned}
& \left|\frac{\partial^{2}}{\partial x_{l}^{2}} \int_{\mathbb{R}^{3}} \Gamma_{\nu}(t, x ; 0, y) a_{i}^{k}(y) d y\right|_{q} \\
& \quad \leq C \frac{1}{t}\left|\int_{\mathbb{R}^{3}}\left(\Gamma_{\nu}(t, x ; 0, y) a_{i}^{k}(y)+\frac{\left(x_{l}-y_{l}\right)^{2}}{t^{2}} \Gamma_{\nu}(t, x ; 0, y) a_{i}^{k}(y)\right) d y\right|_{q} \\
& \quad \leq C t^{-3 / 2+3 / 2 q}|\mathbf{a}|_{3} .
\end{aligned}
$$

By analogous computations, we have

$$
\begin{aligned}
& \left|\frac{\partial^{2}}{\partial x_{l}^{2}} \int_{0}^{t / 2} \int_{\mathbb{R}^{3}} \Gamma_{\nu}(t, x ; s, y) \sum_{j=1}^{3} u_{j}^{k-1}(s, y) \frac{\partial u_{i}^{k-1}}{\partial x_{j}}(s, y) d y\right|_{q} \\
& \quad \leq C C_{0}^{2}\left(|\mathbf{a}|_{3}+|b|_{3}\right)^{2} t^{-3 / 2+3 / 2 q} \int_{0}^{1 / 2}(1-w)^{-7 / 4+3 / 2 q} w^{-3 / 4} d w .
\end{aligned}
$$

The estimation for the terms that involve $\int_{0}^{t / 2}$ are obtained analogously. By other side

$$
\begin{aligned}
\left|\frac{\partial^{2}}{\partial x_{l}^{2}} \int_{t / 2}^{t} \int_{\mathbb{R}^{3}} \Gamma_{\nu}(t, x ; s, y) \sum_{j=1}^{3} u_{j}^{k-1}(s, y) \frac{\partial u_{i}^{k-1}}{\partial x_{j}}(s, y) d y d s\right|_{q} \\
\leq C C_{0}^{2}\left(|\mathbf{a}|_{3}+|b|_{3}\right)^{2} t^{-3 / 2+3 / 2 q} \int_{1 / 2}^{1}(1-w)^{-1 / 2} w^{-2+3 / 2 q} d w \\
\quad+C C_{0}\left(|\mathbf{a}|_{3}+|b|_{3}\right) \int_{1 / 2}^{1}(1-w)^{-1 / 2} w^{-2+3 / 2 q} d w \\
\quad \times \sup _{s \in[t / 2, t]}\left|s^{-3 / 2+3 / 2 q} \frac{\partial^{2} \mathbf{u}^{k-1}}{\partial x_{l}^{2}}(s)\right|_{q} .
\end{aligned}
$$


Analogously

$$
\begin{aligned}
& \left|\frac{\partial^{2}}{\partial x_{l}^{2}} \int_{t / 2}^{t} \int_{\mathbb{R}^{3}} \Gamma_{\nu}(t, x ; s, y) \frac{\partial \pi}{\partial x_{i}} d y d s\right|_{q} \\
& \quad \leq C \int_{t / 2}^{t}(t-s)^{-2}\left(\left|\frac{\partial}{\partial x_{l}}\left(\left(\mathbf{u}^{k-1} \cdot \nabla\right) \mathbf{u}^{k-1}\right)\right|_{q}+\left|\frac{\partial \theta^{k-1} \mathbf{f}}{\partial x_{l}}\right|_{q}\right) d s
\end{aligned}
$$

and, finally,

$$
\begin{aligned}
& \left|\frac{\partial^{2}}{\partial x_{l}^{2}} \int_{t / 2}^{t} \int_{\mathbb{R}^{3}} \Gamma_{\nu}(t, x ; s, y) \theta^{k-1} f_{i} d y d s\right|_{q} \\
& \quad \leq C C_{0}^{2}\left(|\mathbf{a}|_{3}+|b|_{3}\right)^{2} t^{-3 / 2+3 / 2 q} \int_{1 / 2}^{1}(1-w)^{-1 / 2} w^{-2+3 / 2 q} d w .
\end{aligned}
$$

The proof of Lemma 2.4 is a consequence from the above estimative.

Lemma 2.5. Let $\mathbf{a} \in L_{\sigma}^{3}\left(\mathbb{R}^{3}\right), \quad b \in L^{3}\left(\mathbb{R}^{3}\right)$ and $|\mathbf{f}|_{q} \leq C_{0} t^{-1+3 / 2 q}\left(|\mathbf{a}|_{3}+|b|_{3}\right), \quad|\nabla \mathbf{f}|_{q} \leq$ $C_{0} t^{-3 / 2+3 / 2 q}\left(|\mathbf{a}|_{3}+|b|_{3}\right)$. If $M\left(|\mathbf{a}|_{3}+|b|_{3}\right) \leq 1$ for some constant $M$, then for $3 \leq q \leq \infty$, the following estimative are verified uniformly in $k$ :

$$
\begin{gathered}
t^{3 / 2-3 / 2 q}\left|\nabla \pi^{k}\right|_{q} \leq C\left(|\mathbf{a}|_{3}+|b|_{3}\right)^{2} \\
t^{3 / 2-3 / 2 q}\left(\left|\frac{\partial \mathbf{u}^{k}}{\partial t}\right|_{q}+\left|\frac{\partial \theta^{k}}{\partial t}\right|_{q}\right) \leq C\left(|\mathbf{a}|_{3}+|b|_{3}\right)+C\left(|\mathbf{a}|_{3}+|b|_{3}\right)^{2} .
\end{gathered}
$$

Proof. The proof is a consequence of Lemmas 2.2, 2.3, and 2.4, and the following facts

$$
\begin{gathered}
\frac{\partial \mathbf{u}^{k}}{\partial t}=v \triangle \mathbf{u}^{k}-\left(\mathbf{u}^{k-1} \cdot \nabla\right) \mathbf{u}^{k-1}-\nabla \pi^{k}+\theta^{k-1} \mathbf{f} \\
\frac{\partial \theta^{k}}{\partial t}=\chi \triangle \theta^{k}-\mathbf{u}^{k-1} \cdot \nabla \theta^{k-1}
\end{gathered}
$$

The main result in this paper is the following.

Theorem 2.6. Let $\mathbf{a} \in L_{\sigma}^{3}\left(\mathbb{R}^{3}\right), b \in L^{3}\left(\mathbb{R}^{3}\right)$ and $|\mathbf{f}|_{q} \leq C_{0} t^{-1+3 / 2 q}\left(|\mathbf{a}|_{3}+|b|_{3}\right),|\nabla \mathbf{f}|_{q} \leq$ $C_{0} t^{-3 / 2+3 / 2 q}\left(|\mathbf{a}|_{3}+|b|_{3}\right)$. Then, there exists a positive constant $\varepsilon$ such that, if $\left(|\mathbf{a}|_{3}+|b|_{3}\right) \leq$ $\varepsilon$, there exists a unique solution $(\mathbf{u}, \theta)$ for (1.1), which satisfy:

$$
\begin{aligned}
t^{1 / 2-3 / 2 q} \mathbf{u} & \in B C\left([0, \infty) ; L^{q}\left(\mathbb{R}^{3}\right)\right), \\
t^{1 / 2-3 / 2 q} \theta & \in B C\left([0, \infty) ; L^{q}\left(\mathbb{R}^{3}\right)\right), \\
t^{1-3 / 2 q}|\nabla \mathbf{u}| & \in B C\left([0, \infty) ; L^{q}\left(\mathbb{R}^{3}\right)\right), \\
t^{1 / 2-3 / 2 q}|\nabla \theta| & \in B C\left([0, \infty) ; L^{q}\left(\mathbb{R}^{3}\right)\right),
\end{aligned}
$$


for $3 \leq q \leq \infty$ and moreover

$$
\begin{gathered}
t^{3 / 2-3 / 2 q} \sum_{i, j=1}^{3}\left|\frac{\partial^{2} \mathbf{u}}{\partial x_{j} \partial x_{i}}\right| \in B C\left([0, \infty) ; L^{q}\left(\mathbb{R}^{3}\right)\right), \\
t^{3 / 2-3 / 2 q} \sum_{i, j=1}^{3}\left|\frac{\partial^{2} \theta}{\partial x_{j} \partial x_{i}}\right| \in B C\left([0, \infty) ; L^{q}\left(\mathbb{R}^{3}\right)\right), \\
t^{3 / 2-3 / 2 q}|\nabla \pi| \in B C\left([0, \infty) ; L^{q}\left(\mathbb{R}^{3}\right)\right), \\
t^{3 / 2-3 / 2 q} \frac{\partial \mathbf{u}}{\partial t} \in B C\left([0, \infty) ; L^{q}\left(\mathbb{R}^{3}\right)\right),
\end{gathered}
$$

for $3 \leq q \leq \infty$.

Proof. Using Lemma 2.3 with $q=3$, we obtain

$$
\begin{gathered}
\left|\mathbf{u}^{k}\right|_{3}+\left|\theta^{k}\right|_{3} \leq C\left(|\mathbf{a}|_{3}+|b|_{3}\right), \\
\left|\nabla \mathbf{u}^{k}\right|_{3}+\left|\nabla \theta^{k}\right|_{3} \leq C t^{-1 / 2}\left(|\mathbf{a}|_{3}+|b|_{3}\right)
\end{gathered}
$$

then, for $1<p<2$ it is easy to show

$$
\begin{aligned}
\mathbf{u}^{k} & \in L^{\infty}\left(0, \infty ; L^{3}\left(\mathbb{R}^{3}\right)\right), \\
\theta^{k} & \in L^{\infty}\left(0, \infty ; L^{3}\left(\mathbb{R}^{3}\right)\right), \\
\nabla \mathbf{u}^{k} & \in L_{\mathrm{loc}}^{p}\left(0, \infty ; L^{3}\left(\mathbb{R}^{3}\right)\right), \\
\nabla \theta^{k} & \in L_{\mathrm{loc}}^{p}\left(0, \infty ; L^{3}\left(\mathbb{R}^{3}\right)\right) .
\end{aligned}
$$

Let $q$ and $q^{*}$ such that $1 / q+1 / q^{*}=1$ and we consider the following estimative:

$$
\begin{gathered}
\sup _{|\mathbf{v}|_{W_{0}^{1, q^{*}}=1}}\left|\left\langle\Delta \mathbf{u}^{k}, \mathbf{v}\right\rangle\right| \leq \sup _{|\mathbf{v}|_{W_{0}^{1, q^{*}}=1}}\left|\nabla \mathbf{u}^{k}\right|{ }_{q}|\nabla \mathbf{v}|_{q^{*}} \leq\left|\nabla \mathbf{u}^{k}\right|_{q^{\prime}}, \\
\sup _{|\mathbf{v}|_{W_{0}^{1, q^{*}=1}}}\left|\left\langle\left(\mathbf{u}^{k-1} \cdot \nabla\right) \mathbf{u}^{k-1}, \mathbf{v}\right\rangle\right| \leq\left.\left.|| \mathbf{u}^{k-1}\right|^{2}\right|_{q^{\prime}} \\
\sup _{|\mathbf{v}|_{W_{0}^{1, q^{*}=1}}}\left|\left\langle\nabla \pi^{k}, \mathbf{v}\right\rangle\right| \leq\left|\pi^{k}\right|_{q^{*}}
\end{gathered}
$$

Now, using Hölder and Sobolev inequalities, we have

$$
\begin{aligned}
\sup _{|\mathbf{v}|_{W_{0}^{1, q^{*}}}}\left|\left\langle\theta^{k-1} \mathbf{f}, \mathbf{v}\right\rangle\right| & \leq C \sup _{|\mathbf{v}|_{W_{0}^{1, q^{*}}=1}}\left|\theta^{k-1} \mathbf{f}\right|_{3 q /(q+3)}|\nabla \mathbf{v}|_{q^{*}} \\
& =C\left|\theta^{k-1} \mathbf{f}\right|_{3 q /(q+3)^{*}}
\end{aligned}
$$

Thus, by using Lemma 2.4 together with Sobolev and Hölder inequalities, we obtain

$$
\left|\frac{\partial \mathbf{u}^{k}}{\partial t}\right|_{W^{-1, q}} \leq\left|\nabla \mathbf{u}^{k}\right|_{q}+C\left|\mathbf{u}^{k-1}\right|_{3}\left|\nabla \mathbf{u}^{k-1}\right|_{q}+C\left|\theta^{k-1}\right|_{3}|\mathbf{f}|_{q},
$$


consequently,

$$
\left|\frac{\partial \mathbf{u}^{k}}{\partial t}\right|_{W^{-1, q}} \leq C t^{-1+3 / 2 q} .
$$

Analogously the following inequality can be proved for the temperature $\theta^{k}$

$$
\left|\frac{\partial \theta^{k}}{\partial t}\right|_{W^{-1, q\left(\mathbb{R}^{3}\right)}} \leq C t^{-1+3 / 2 q} .
$$

Therefore, for $1<r<2 q /(2 q-3)$, we have

$$
\begin{gathered}
\frac{\partial \mathbf{u}^{k}}{\partial t} \in L_{\mathrm{loc}}^{r}\left(0, \infty ; W^{-1, q}\left(\mathbb{R}^{3}\right)\right), \\
\frac{\partial \theta^{k}}{\partial t} \in L_{\mathrm{loc}}^{r}\left(0, \infty ; W^{-1, q}\left(\mathbb{R}^{3}\right)\right) .
\end{gathered}
$$

By using the compact embedding of $W^{1,3}\left(\mathbb{R}^{3}\right)$ on $L_{\text {loc }}^{3}\left(\mathbb{R}^{3}\right)$ and the Compactness Theorem in [11, Cap. 3], we obtain that there exists $(\mathbf{u}, \theta)$ such that

$$
\begin{array}{ll}
\mathbf{u}^{k} \longrightarrow \mathbf{u} & \text { in } L_{\mathrm{loc}}^{2}\left(0, \infty ; L_{\mathrm{loc}}^{3}\left(\mathbb{R}^{3}\right)\right) \text { strongly, } \\
\theta^{k} \longrightarrow \theta & \text { in } L_{\mathrm{loc}}^{2}\left(0, \infty ; L_{\mathrm{loc}}^{3}\left(\mathbb{R}^{3}\right)\right) \text { strongly. }
\end{array}
$$

Now, using the standard arguments, it is easily to show that $(\mathbf{u}, \theta)$ is a unique solution of (1.1) (see [5]).

\section{Acknowledgments}

The authors has been partially supported by D.G.E.S. and M.C. y T. (Spain), Project BFM2003-06446. M.A. Rojas-Medar is partially supported by CNPq-Brazil, Grant 301354/03-0.

\section{References}

[1] R. A. Adams, Sobolev Spaces, Pure and Applied Mathematics, vol. 65, Academic Press, New York, 1975.

[2] J. R. Cannon and E. DiBenedetto, The initial value problem for the Boussinesq equations with data in $L^{p}$, Approximation Methods for Navier-Stokes Problems (Proc. Sympos., Univ. Paderborn, Paderborn, 1979), Lecture Notes in Math., vol. 771, Springer, Berlin, 1980, pp. 129-144.

[3] Y. Giga, Solutions for semilinear parabolic equations in $L^{p}$ and regularity of weak solutions of the Navier-Stokes system, J. Differential Equations 62 (1986), no. 2, 186-212.

[4] C. He and L. Hsiao, The decay rates of strong solutions for Navier-Stokes equations, J. Math. Anal. Appl. 268 (2002), no. 2, 417-425.

[5] T. Hishida, Existence and regularizing properties of solutions for the nonstationary convection problem, Funkcial. Ekvac. 34 (1991), no. 3, 449-474.

[6] D. D. Joseph, Stability of Fluid Motions. Vol. I, Springer Tracts in Natural Philosophy, vol. 27, Springer, Berlin, 1976.

[7] _ Stability of Fluid Motions. Vol. II, Springer Tracts in Natural Philosophy, vol. 28, Springer, Berlin, 1976. 


\section{Boussinesq equations}

[8] O. A. Ladyzhenskaya, The Mathematical Theory of Viscous Incompressible Flow, Mathematics and Its Applications, vol. 2, Gordon and Breach, New York, 1969.

[9] J.-L. Lions, Quelques méthodes de résolution des problèmes aux limites non linéaires, Dunod, Paris, 1969.

[10] M. A. Rojas-Medar and S. A. Lorca, The equations of a viscous incompressible chemical active fluid. I. Uniqueness and existence of the local solutions, Rev. Mat. Apl. 16 (1995), no. 2, 5780 .

[11] The equations of a viscous incompressible chemical active fluid. II. Regularity of solutions, Rev. Mat. Apl. 16 (1995), no. 2, 81-95.

[12] R. Temam, Navier-Stokes Equations, Revised ed., Studies in Mathematics and Its Applications, vol. 2, North-Holland, Amsterdam, 1979.

Francisco Guillén González: Universidad de Sevilla, Facultad de Matemáticas, 41012 Sevilla, Spain E-mail address: guillen@us.es

Márcio Santos da Rocha: DM-CCE-UEL, Londrina-PR, Brazil

E-mail address: marcio@uel.br

Marko Rojas Medar: DMA-IMECC-UNICAMP, CP 6065, 13083-970, Campinas-SP, Brazil

E-mail address: marko@ime.unicamp.br 


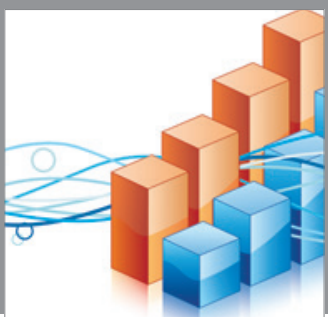

Advances in

Operations Research

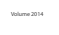

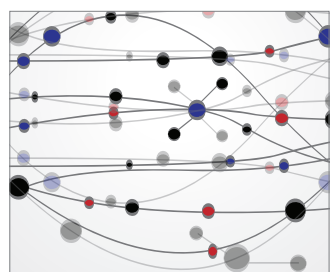

\section{The Scientific} World Journal
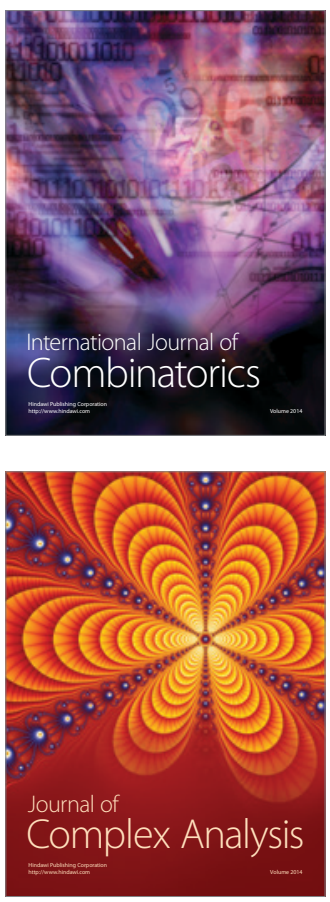

International Journal of

Mathematics and

Mathematical

Sciences
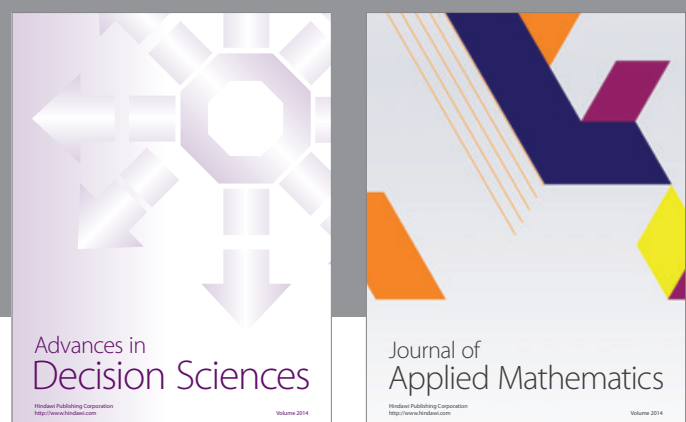

Journal of

Applied Mathematics
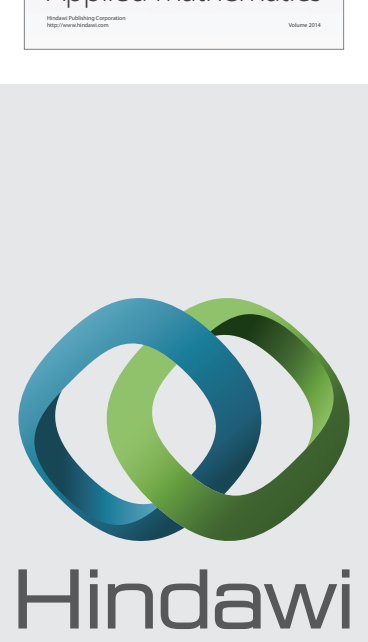

Submit your manuscripts at http://www.hindawi.com
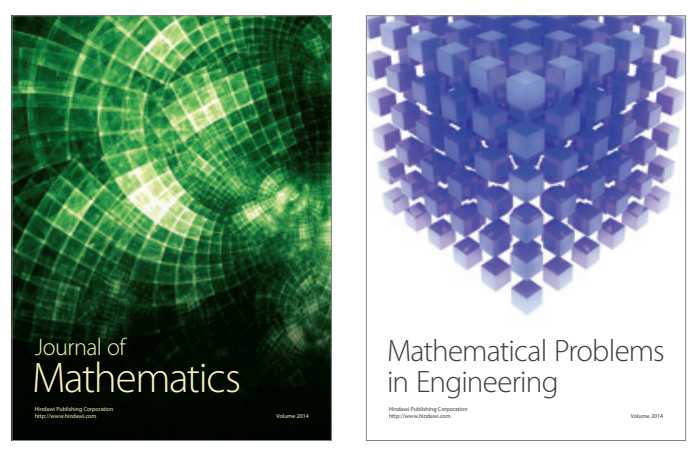

Mathematical Problems in Engineering
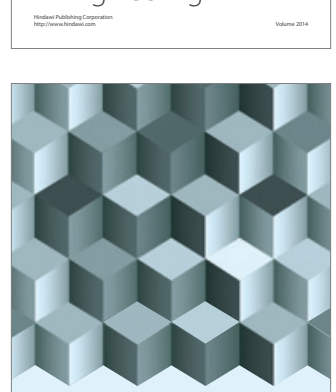

Journal of

Function Spaces
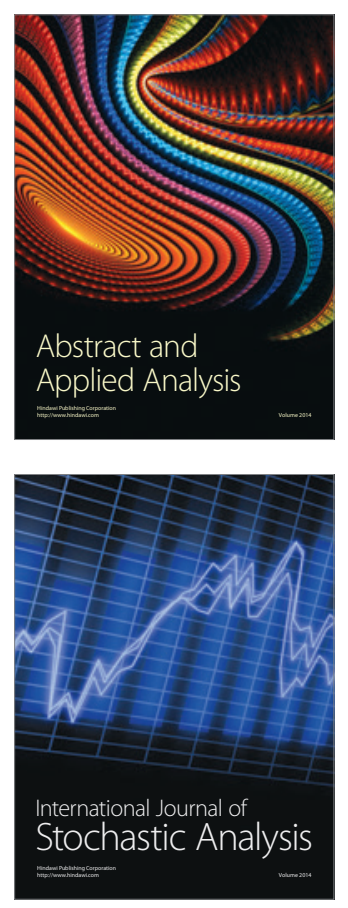

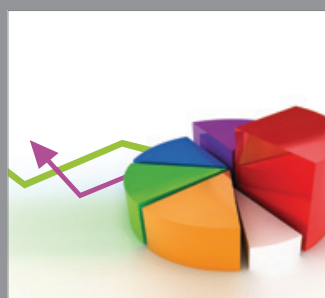

ournal of

Probability and Statistics

Promensencen
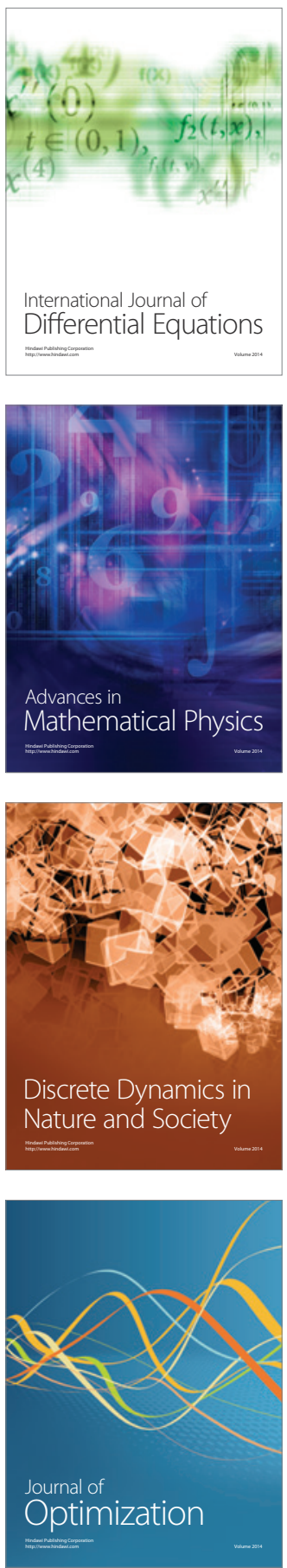